EPJ Web of Conferences 41, 02009 (2013)

DOI: $10.1051 /$ epjconf/20134102009

CC Owned by the authors, published by EDP Sciences, 2013

\title{
Ultrafast Nonlinear Double Excitations of He in Intense EUV FEL Fields
}

\author{
M. Fushitani ${ }^{1}$, Y. Hikosaka ${ }^{2}$, A. Matsuda ${ }^{1}$, C.-N. Liu ${ }^{3}$, T. Morishita ${ }^{4}$, E. Shigemasa ${ }^{5}$, and \\ A. Hishikawa ${ }^{1}$ \\ ${ }^{1}$ Department of Chemistry, Graduate School of Science, Nagoya University, Nagoya, Aichi 464- \\ 8602, Japan \\ ${ }^{2}$ Department of Environmental Science, Niigata University, Niigata, Niigata 950-2181, Japan \\ ${ }^{3}$ Department of Physics, Fu-Jen Catholic University, Taipei 24205, Taiwan \\ ${ }^{4}$ Department of Engineering Science, University of Electro-Communications, Chofu, Tokyo 182- \\ 8585, Japan \\ ${ }^{5}$ Institute for Molecular Science, National Institutes of Natural Sciences, Okazaki, Aichi 444-8585, \\ Japan
}

\begin{abstract}
Three-photon double excitation of He in intense EUV FEL fields is studied by the shot-by-shot photoelectron spectroscopy, revealing the enhancement by resonances to the doubly excited states converging to the $\mathrm{He}^{+} N=3$ level.
\end{abstract}

\section{Introduction}

The recent advances in free electron laser (FEL) technology have enabled us to generate intense ultrashort laser fields in the extreme ultraviolet (EUV). Atoms exposed to intense laser fields typically undergo multiphoton multiple ionization. However, unlike a non-linear process in the optical regime where only the valence electrons are involved, inner-valence or inner-core electrons can also participate in multiphoton process in EUV due to the large photon energy, which may open a new ionization channel. In our previous studies [1], we have demonstrated that single-shot photoelectron spectroscopy provides detailed information on the multi-photon processes of simple atoms and molecules utilizing the inherent fluctuation of FEL pulses. Here, we apply the single shot photoelectron spectroscopy to double excitation of He in ultrashort pulse duration of EUV-FEL pulses [2]. The present study provides a route towards an efficient creation of doubly excited states of $\mathrm{He}$, making it possible to investigate dynamics of strongly correlated electrons in real time.

\section{Experimental}

We carried out the experiment by using EUV-FEL pulses from the SCSS Test Accelerator at RIKEN. The nominal center wavelength can be tuned in the range of 50-61 nm and the typical pulse energy is $\sim 10 \mu \mathrm{J}$. EUV-FEL pulses $\left(51.0 \mathrm{~nm}, 5 \mathrm{TW} / \mathrm{cm}^{2}, 20 \mathrm{~Hz}\right)$ were focused to sample gas in the interaction region about $25 \mu \mathrm{m}$ in diameter by elliptical and cylindrical mirrors. The intensity of FEL pulses was controlled and monitored by using an Ar gas attenuator installed in the upper stream of the FEL beam line.

This is an Open Access article distributed under the terms of the Creative Commons Attribution License 2.0, which permits unrestricted use, distribution, and reproduction in any medium, provided the original work is properly cited. 
Electrons emitted by photoionization were detected with a collection efficiency of $4 \pi$ solid angle by using a magnetic bottle type photoelectron spectrometer. The high collection efficiency allowed us to keep the ionization event rate low enough to avoid a space charge effect and to measure photoelectron spectra on the shot-to-shot basis [1,2]. The photoelectron spectroscopy is powerful in elucidating the non-linear processes in EUV-FEL fields, because (i) electronic states involved in the ionization process can be identified directly from the photoelectron energy and (ii) the spectral properties of fluctuating FEL pulses can be evaluated on the shot-by-shot basis. Electron energy was calibrated by comparing high-resolution photoelectron spectra of $4 d$ Xe Auger electrons [3] and atomic lines by autoionization of oxygen via $\mathrm{O}_{2}{ }^{*}$ dissociation [4], photoinduced by the second and third harmonics of FEL at $51 \mathrm{~nm}$. A typical energy resolution $\Delta E$ of the spectrometer was obtained to be $E / \Delta E=30$ for $E<100 \mathrm{eV}$.

Pump-probe experiments were performed using optical laser pulses delivered from a Ti:Sa laser system with a frequency converter unit, which were irradiated to a sample gas at an angle of $\sim 1^{\circ}$ with respect to the EUV-FEL pulse. The time delay between the EUV-FEL and optical laser pulses was controlled by a delay unit system (CANDOX) with the minimum step of 1 ps.

\section{Results and discussion}

Figure 1(a) shows photoelectron spectra of He by FEL $(51.0 \mathrm{~nm})$ for three different FEL intensities $\left(I_{0}=5 \mathrm{TW} / \mathrm{cm}^{2}\right)$. Doublet peaks seen at 10.8 and $12.2 \mathrm{eV}$ are due to photoelectrons of $\mathrm{Xe} 5 p^{-1}$ spinorbit levels which was used for evaluation of the FEL wavelength. Besides Xe peaks, the most prominent peak observed at $6.9 \mathrm{eV}$ in Fig. 1(a) was assigned to photoelectrons formed by threephoton ionization to electronically excited $(N=2)$ state of $\mathrm{He}^{+}$, whose photoelectron yield shows a nonlinear dependence upon the FEL intensity. The energies of the other peaks at 24 and $48 \mathrm{eV}$ correspond to the two and three-photon $(N=1)$ processes, but these are mostly due to the second and third FEL harmonics respectively, as they show only weak dependence on the FEL intensity. After the subtraction of the background signals due to the harmonics, it was found that the three-photon ionization (observed at 6.9 and $48 \mathrm{eV}$ ) is $>20$ times more efficient than the two-photon process (observed at $24 \mathrm{eV}$ ) at the FEL intensity of $I_{0}=5 \mathrm{TW} / \mathrm{cm}^{2}$

The origin of the three-photon enhancement is clear in an expanded view of the peak at $6.9 \mathrm{eV}$ in Fig. 1(b). Also shown are the results of the single-shot analysis, where each photoelectron spectrum is sorted according to the FEL mean photon energies determined by the Xe photoelectron peaks and then averaged within narrow photon energy ranges that divide the whole photon energy range (23.95-24.17 eV) into 5 segments. The shot-by-shot photoelectron spectra show that the energy positions of the observed fine peaks do not shift by the changes of the center photon energy. This finding clearly shows that some excited states in the neutral He autoionizing manifold are in threephoton resonance from the neutral ground state. Indeed, there are doubly excited states converging to $\mathrm{He}^{+} N=3$ state in the $70 \mathrm{eV}$ energy region [5] as shown in Fig. 1(c). The result demonstrates that the double excitation plays an important role in multiphoton absorption in the EUV region.

The experimental results were simulated by solving the time-dependent Schrödinger equation for two-electrons of He whose correlations were taken into account by the hyperspherical method [2]. The simulated results reproduce the experimental spectra in Fig. 1(b), indicating that the substructure is formed by double excitation where one electron is excited to $N=3$ and the other electron is to $n=4$ 8. Because of these double excitations, three-photon absorption predominantly proceeds compared to two-photon absorption.

Figure 1(d) shows photoelectron spectrum measured with an UV laser $(250 \mathrm{~nm})$ pulse, which was introduced 50 ps after the EUV FEL pulse. From the electron energy, the peaks were assigned to electrons produced from Rydberg states of neutral He with $n=4-8$. Resonances with these Rydberg states contribute to the relative enhancement of the three-photon process, because of a poor overlap integral between the diffuse Rydberg state and the highly oscillatory wavefunction of the highenergy ionization continuum, which results in the relative suppression of the two-photon process against the three-photon process. 

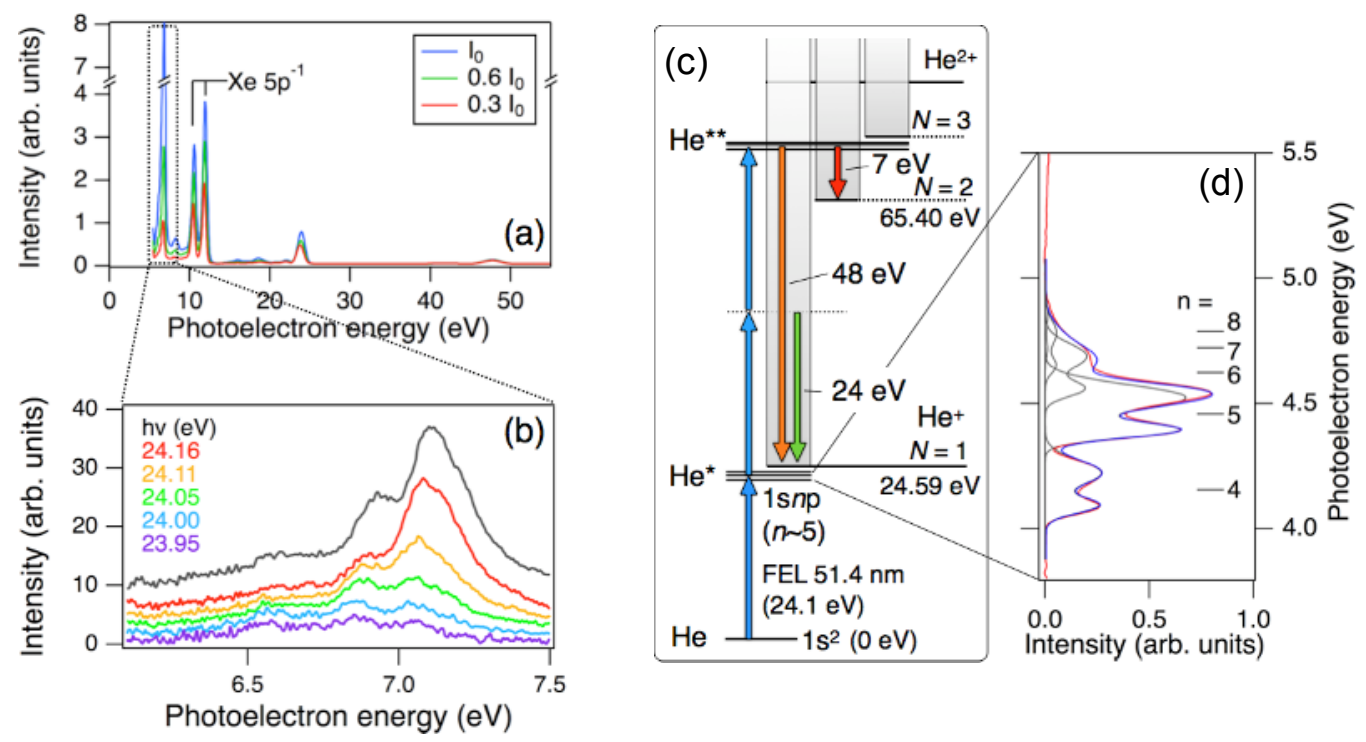

Fig. 1. (a) Photoelectron spectra of He for three different intensities of EUV FEL at $51.0 \mathrm{~nm}$. (b) Photon energy dependence of photoelectron peak around $6.9 \mathrm{eV}$. (c) Relevant energy levels for multiphoton ionization of He. (d) Photoelectron spectra of He obtained by two-color two-photon ionization with FEL at $51.0 \mathrm{~nm}$ and $\mathrm{UV}$ laser at $250 \mathrm{~nm}$.

\section{Summary}

We have demonstrated that ultrafast double excitations of He take place by non-linear multi-photon absorption of intense EUV FEL. The intermediate Rydberg states contribute to the enhancement of the multi-photon process by trapping the first electron driven by the laser field in the inactive states for the ionization. The present study provide how to prepare doubly excited states in an efficient way, making it possible to investigate details of double excitation process in real time.

We are grateful to the staff in the SCSS facility and XFEL project head office for their support of our experiment.

\section{References}

1. Y. Hikosaka, M. Fushitani, A. Matsuda, et al., Phys. Rev. Lett. 105, 133001 (2010)

2. A. Hishikawa, M. Fushitani, Y. Hikosaka, et al., Phys. Rev. Lett. 107, 243003 (2011)

3. T.X. Carroll, J.D. Bozek, E. Kukk, et al., J. Electron Spectrosc. Relat. Phenom. 125, 127 (2002)

4. A.A. Wills, A.A. Cafolla, and J. Comer, J. Phys. B 24, 3989 (1991)

5. M. Domke, K. Schulz, G. Remmers, G. Kaindl, and D. Wintgen, Phys. Rev. A 53, 1424 (1996) 